\title{
Acceleration of ligamentization and osseointegration processes after anterior cruciate ligament reconstruction with autologous tissue-engineered polyethylene terephthalate graft
}

\author{
Jiangyu Cai ${ }^{1,2 \#}, J^{\prime}$ juie Xu ${ }^{1 \#}$, Yuhao Kang ${ }^{1}$, Yufeng Li ${ }^{1}$, Liren Wang ${ }^{1}$, Xiaoyu Yan ${ }^{1}$, Jia Jiang', Jinzhong Zhao ${ }^{1}$ \\ ${ }^{1}$ Department of Sports Medicine, Shanghai Jiao Tong University Affiliated Sixth People's Hospital, Shanghai, China; ${ }^{2}$ State Key Laboratory of \\ Molecular Engineering of Polymers, Fudan University, Shanghai, China \\ Contributions: (I) Conception and design: J Zhao, J Cai, J Xu; (II) Administrative support: X Yan, J Jiang, J Zhao; (III) Provision of study materials \\ or patients: J Cai, J Xu; (IV) Collection and assembly of data: Y Kang, L Wang; (V) Data analysis and interpretation: J Xu, Y Li; (VI) Manuscript \\ writing: J Cai, J Xu; (VII) Final approval of manuscript: All authors. \\ \#These authors contributed equally to this work. \\ Correspondence to: Jinzhong Zhao. Department of Sports Medicine, Shanghai Jiao Tong University Affiliated Sixth People's Hospital, 600 Yishan \\ Road, Shanghai 200233, China. Email: jzzhao@sjtu.edu.cn.
}

Background: Despite the advantages of excellent mechanical properties for rapid return to sports and early rehabilitation after anterior cruciate ligament (ACL) reconstruction with polyethylene terephthalate (PET) artificial ligament, the graft failure rate during long-term follow-up is relatively high due to poor graft-host incorporation. The purpose of the present study was to investigate the effect of autologous tissue-engineered PET (ATE-PET) grafts on osseointegration and ligamentization after ACL reconstruction.

Methods: Forty-eight New Zealand white rabbits were randomly divided into PET group (n=24) and ATEPET group (n=24). In the ATE-PET group, the rabbits initially underwent subcutaneous implantation of the PET ligament. Two weeks later, unilateral ipsilateral ACL reconstruction was performed using an ATEPET graft. In the PET group, the rabbits underwent ACL reconstruction using PET grafts as controls. Macroscopic observation, micro-computed tomography, histological and immunofluorescent staining, and biomechanical tests were conducted to evaluate the effects at 4 and 12 weeks postoperatively.

Results: The ATE-PET graft was highly pre-vascularized with myofibroblast aggregation after two weeks of subcutaneous implantation. With regard to the intraosseous part of the graft, the ATE-PET group had significantly higher bone mineral density and bone volume/total volume ratio at 12 weeks. Histologically, the width of the interface between the graft and bone was smaller. Regarding the intra-articular part, thicker tissue coverage with a glossy appearance was observed in the ATE-PET group at 12 weeks on macroscopic observation. Histological staining also showed more collagen fibers grew in the grafts with fewer inflammatory reactions of the ATE-PET group at both 4 and 12 weeks. Immunofluorescently, both $\alpha$-SMA-positive vessels and $\alpha$-SMA-positive myofibroblasts were found to be significantly greater around the graft in the ATE-PET group at 4 weeks and markedly declined at 12 weeks. Moreover, the ATE-PET group presented significantly greater failure load and stiffness than the PET group at 12 weeks $(53.7 \pm 5.4 v s$. $42.5 \pm 4.5 \mathrm{~N}, \mathrm{P}<0.01 ; 12.9 \pm 3.0$ vs. $9.8 \pm 1.3 \mathrm{~N} / \mathrm{mm}, \mathrm{P}=0.04)$.

Conclusions: The ATE-PET artificial ligament with pre-vascularization and myofibroblast aggregation could effectively accelerate intra-articular graft ligamentization and intraosseous graft osseointegration, thus enhancing the biomechanical properties after ACL reconstruction in a rabbit model.

Keywords: Anterior cruciate ligament reconstruction; autologous tissue engineering; artificial ligament; ligamentization; osseointegration

Submitted Dec 18, 2020. Accepted for publication Mar 05, 2021.

doi: 10.21037/atm-20-8048

View this article at: http://dx.doi.org/10.21037/atm-20-8048 


\section{Introduction}

Anterior cruciate ligament (ACL) rupture is a very common pathology in the field of sports medicine. It causes great instability, loss of motion, and further degenerative diseases of the knee (1-3). ACL reconstruction is considered as the standard treatment for ACL rupture. Autografts and allografts are main choices for surgical ACL reconstruction in clinical practice (4). However, these grafts have some drawbacks, such as donor site morbidity of autografts, potential disease transmission, and allergic reactions derived from allografts $(5,6)$. Moreover, the autografts and allografts undergo a period of biological transformation. The mechanical strength of the graft at the early healing phase becomes significantly lower than that at the time of implantation. This compromises the early and intensive rehabilitation phase, which could lead to graft failure after surgery.

The Ligament Augmentation Reinforcement System (LARS) is the most commonly used synthetic device in ACL reconstruction. It consists of fibers made of polyethylene terephthalate (PET) (7). Despite the advantages of high tensile strength for rapid return to sports and early rehabilitation postoperatively, LARS leads to a relatively high rate of graft failure in the medium- and long-term follow-up (8). Recently, studies showed that the failure rate of ACL reconstruction with LARS was $33.3 \%$ at a mean follow-up of 3.9 years and $31 \%$ at a mean follow-up of 9.5 years $(8,9)$. There are several possible reasons for the high failure rates of LARS devices. These include issues related to surgical techniques, such as inappropriate bone tunnel or screw positions (3). There are also mechanical factors, including inadequate fiber abrasion resistance or flexural and rotational fatigue (10). In addition, biological factors, such as foreign body synovitis or poor biological integration, may account for the high failure rates (8). Among these reasons, poor biological integration in the bone tunnels and articular cavity is thought to be the primary issue (11). Therefore, it is imperative to improve the biocompatibility of PET, and to accelerate the osseointegration of the intraosseous part and the ligamentization of the intra-articular part of LARS devices after ACL reconstruction.

Several studies have focused on the surface modification of PET artificial ligaments with bioactive substances, such as calcium phosphate, silk fibroin, and bioglass, for ACL reconstruction (12-14). However, the weak adhesive strength of these substances to PET and the potential biotoxicity of modification techniques prevent their implementation in clinical practice (15). Moreover, ACL remnant preservation (16), enhancement with periosteum patch (17), and combination with autologous tendon (18) have been reported to be applied for ACL reconstruction with PET artificial ligaments. Although these strategies are clinically feasible, the incorporation of the PET graft and host is still not perfect after ACL reconstruction. In recent years, multiple strategies based on tissue engineering have been applied to form a functional microvasculature with the host, thus improving graft or scaffold incorporation to deliver promising results $(19,20)$. Rapid pre-vascularization based on tissue engineering is very important for the survival of transplanted scaffolds and grafts. It has been reported that an in vivo engineered pre-vascularization strategy can utilize the body itself as a bioreactor to form new blood vessels within the implanted scaffolds for skin and bone tissue regeneration, which is promising for clinical applications $(21,22)$.

Inspired by this strategy, in the present study, the PET ligament was implanted subcutaneously and underwent in vivo autologous tissue engineering. We aimed to investigate the effects of autologous tissue-engineered PET (ATE-PET) grafts on osseointegration and ligamentization after ACL reconstruction in an animal study. It was hypothesized that the pre-vascularized PET graft with ATE preprocess might have positive effects on the osseointegration and ligamentization processes, thus improving the biomechanical properties of the reconstructed ACL. The findings from our study may inform the treatment of ACL rupture in clinical practice.

We present the following article in accordance with the ARRIVE reporting checklist (available at http://dx.doi. org/10.21037/atm-20-8048).

\section{Methods}

\section{Study design and procedure}

The PET ligament was obtained from LARS. Forty-eight skeletally mature male New Zealand white rabbits (weight, $2.7-3.5 \mathrm{~kg}$ ) were randomly divided into the PET group $(\mathrm{n}=24)$ and ATE-PET group $(\mathrm{n}=24)$. Randomization was achieved by using online random number generators. All animal experiments were approved by the Animal Care and Experiment Committee of Shanghai Jiao Tong University (No.: 201018) and were conducted in accordance with the local animal care guidelines. Under standardized environmental conditions, the rabbits were housed in separate cages with free access to water and food. The room temperature was maintained at $25 \pm 2{ }^{\circ} \mathrm{C}$. 

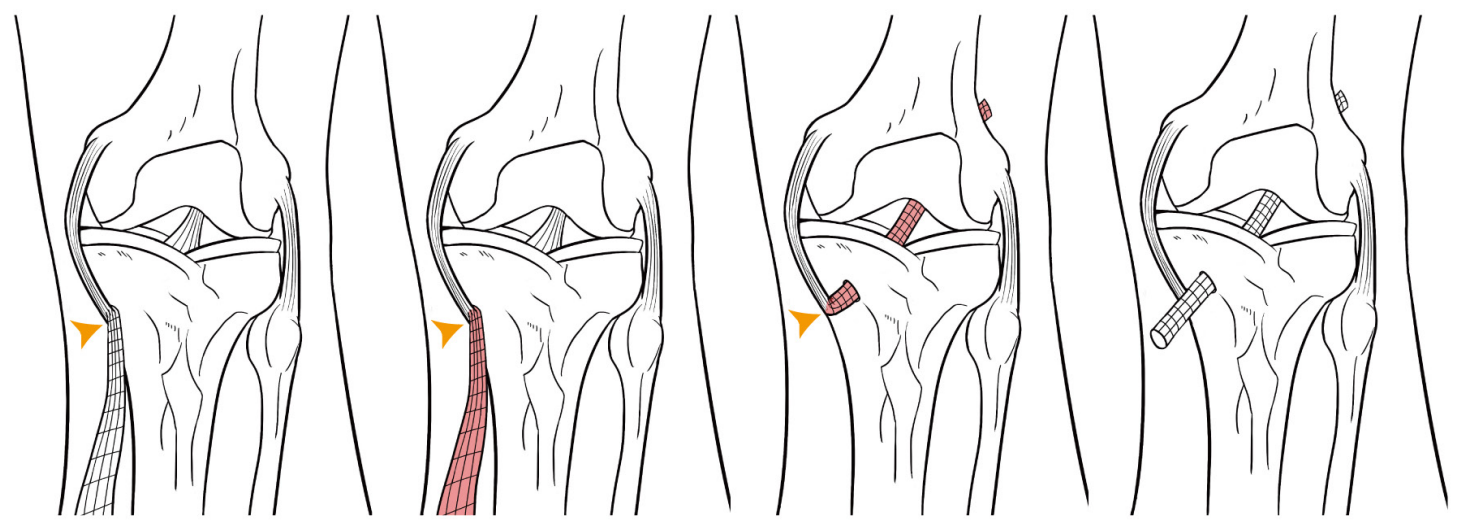

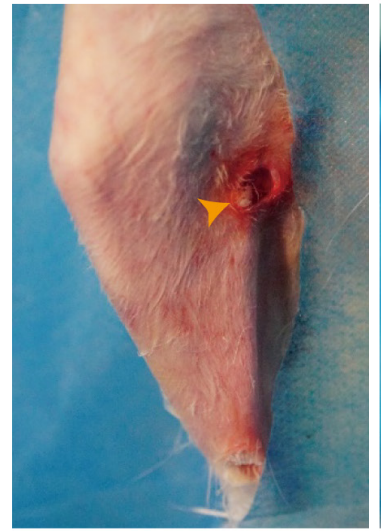

Sub-implantation

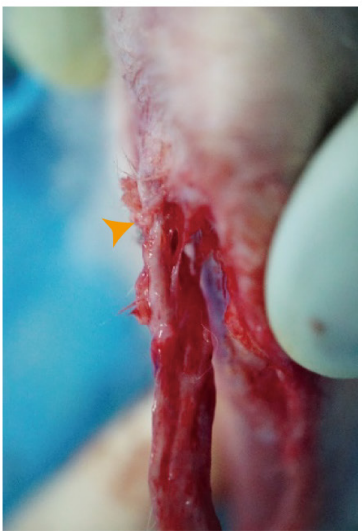

ATE

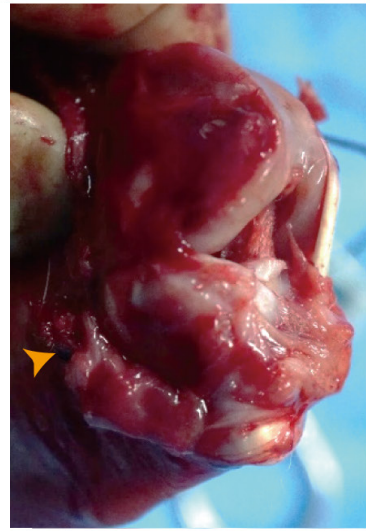

ACLR with ATE-PET

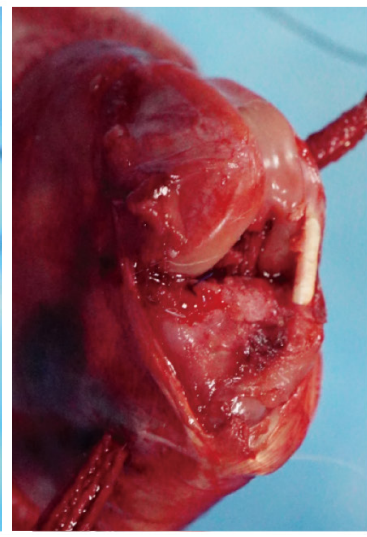

ACLR with PET

Figure 1 Schematic diagram of ACL reconstruction with subcutaneously implanted ATE-PET or pure PET graft. Yellow arrowheads indicate the proximal end of ATE-PET graft. Sub-implantation, subcutaneous implantation; ATE, autologous tissue-engineered; PET, polyethylene terephthalate; ACLR, ACL reconstruction.

In the ATE-PET group, all rabbits initially underwent a minimally invasive procedure of subcutaneous implantation with PET ligaments (Figure 1). Specifically, after anesthesia was induced by intramuscular injection of $0.8 \mathrm{~mL}$ of xylazine hydrochloride and $0.8 \mathrm{~mL}$ of diazepam, a minimal incision was made on the medial side of the proximal tibia. One end of the PET ligament was sutured to the tibial insertion of the medial collateral ligament (MCL), and the entire ligament was implanted subcutaneously with blunt separation of tissues. Two weeks later, unilateral ipsilateral ACL reconstruction was performed using an ATE-PET graft. Briefly, after a medial parapatellar arthrotomy was created, the patella was dislocated outward to expose the knee joint and the ACL. The native ACL was removed from the insertion sites, and 2.5-mm-diameter tibial and femoral tunnels were established along the original ACL footprint with an orthopedic drill. Then, the ATEPET graft was carefully blunted from the distal end using a designed tendon extractor of 2.5 -mm-diameter with the proximal one fixed on the MCL. Following this, the distal end of the graft was pulled into the tibial tunnel and passed through the femoral tunnel using a PDS-II suture (Ethicon, Puerto Rico, USA). Both ends of the graft were sutured with the adjacent periosteum and soft tissue, and the wound was closed layer by layer. In the PET group, all rabbits underwent ACL reconstruction with a PET graft of the same diameter $(2.5 \mathrm{~mm})$, without subcutaneous implantation preprocesses as controls.

Postoperatively, the animals were returned to their cages without immobilization. Penicillin $(400,000 \mathrm{U}$, muscle injection) was administered daily for 3 days. Twelve rabbits from each group were sacrificed at each time point (4 or 12 weeks) after ACL reconstruction for further evaluations, including macroscopic observation, micro-computed tomography (CT) analysis, histological assessment, immunofluorescence imaging, and biomechanical testing. 


\section{Micro-CT analysis}

After sacrificing the rabbits at 4 and 12 weeks, the grafttibia complex samples ( $\mathrm{n}=6$ per group and time point) were fixed in $10 \%$ formalin for $48 \mathrm{~h}$. The samples were scanned perpendicular to the long bone axis at a spatial resolution of $18 \mu \mathrm{m}(1 \mathrm{~mm}$ aluminum filter, $65 \mathrm{KV}, 378 \mu \mathrm{A})$ using a Skyscan 1176 micro-CT imaging system (Bruker, Kontich, Belgium). To analyze the bone mineral density (BMD) and bone volume/total volume ratio (BV/TV) of the proximal end of the tibial tunnel or the distal end of the femoral tunnel, cylindrical regions of interest $(4 \mathrm{~mm}$ in diameter and $4 \mathrm{~mm}$ in height) were applied as previously reported (23).

\section{Histological assessment}

After the micro-CT scan, the samples were decalcified at room temperature in $10 \%$ ethylenediaminetetraacetic acid for 4 weeks. After dehydration and embedding in paraffin, the samples were sectioned at a thickness of $5 \mu \mathrm{m}$ perpendicular to the longitudinal axis of the intraosseous part of the graft using a microtome (SM2500; Leica, Nussloch, Germany). Furthermore, the samples of subcutaneously implanted ATE-PET ligament after 2 weeks and the intra-articular part of the graft were also sectioned perpendicular to the longitudinal axis. All sections were stained with hematoxylin-eosin (H\&E) and Masson trichrome and were observed under an inverted light microscope (IX71SBF-2, Olympus Co., Japan). Digital images were obtained using a DP Manager (Olympus Optical Co., Japan). Further semiquantitative analyses of the intraosseous and intra-articular sections were performed using Image-Pro Plus 6.0 software (Media Cybernetics, Silver Spring, MD, USA) by two independent observers blinded to the intervention and group allocation. H\&E staining was used to evaluate intraosseous graft-to-bone integration by measuring the average interface width between the bone and graft $(11,24)$. Each section was divided into four quadrants to determine the interface width, which was measured as the distance between the edge of the bone tunnel and the outer graft. Four separate measurements were made in each quadrant for a total of 16 measurements for each specimen. The interface width was then determined by averaging these values, and a smaller interface width indicated better graft-to-bone integration. Moreover, Masson's trichrome staining was performed to localize the collagen distribution in the regenerated tissues of the intra-articular region (25). In this assay, blue staining indicated the presence of collagen fibers. The collagen volume fraction (CVF) was calculated as follows: CVF (\%) $=$ collagen area/total area $\times 100 \%$. Each section was divided into four quadrants, and data were averaged from a total of four measurements for each specimen.

\section{Immunofluorescence imaging}

Immunofluorescence staining against $\alpha$-SMA was performed to detect vascular smooth muscle cells (26) and myofibroblasts (27), according to the manufacturer's protocol. Briefly, after dewaxing, rehydration, and antigen retrieval, the sections were blocked with $2 \%$ bovine serum albumin in phosphate buffer saline (PBS) for $1 \mathrm{~h}$. Sections were incubated with anti- $\alpha$-SMA antibody (Arigo Biolaboratories, Taiwan, China) overnight at $4{ }^{\circ} \mathrm{C}$ and then with goat anti-mouse secondary antibody labeled with Alexa 488 (Molecular Probes, Invitrogen, Carlsbad, CA) for $1 \mathrm{~h}$ at $37^{\circ} \mathrm{C}$. At the end of the incubation period, the sections were washed with PBS and counterstained with DAPI (Life Technologies, CA, USA). Images were observed using fluorescence microscopy (IX53, Olympus) for further semiquantitative analysis. For each section of the intraosseous or intra-articular part, six random fields of view (FOV) around the graft were randomly selected, and $\alpha$-SMA positive vessels and myofibroblasts were counted by two independent observers using Image-Pro Plus 6.0. $(27,28)$. The positive ellipse was an $\alpha$-SMA-positive vessel, and the positive spindle cell was an $\alpha$-SMA-positive myofibroblast.

\section{Biomechanical test}

The graft-tibia complex samples ( $\mathrm{n}=6$ per group and time point) were harvested and prepared for biomechanical testing immediately after sacrifice. After the soft tissues, except for the graft, were carefully removed from the samples, mechanical testing was performed using an electronic universal material testing system machine (AGS-X, Shimadzu, Co., Japan). After a preloading of $1 \mathrm{~N}$, a load displacement rate of $5 \mathrm{~mm} / \mathrm{min}$ was applied to the samples until the graft was ruptured or pulled out of the bone tunnel. The ultimate failure load and stiffness were recorded based on the load-deformation curves.

\section{Statistical analysis}

Continuous data were presented as the mean \pm standard deviation. The Shapiro-Wilk test was used for the normality test, and the Levene test was used for homogeneity of 

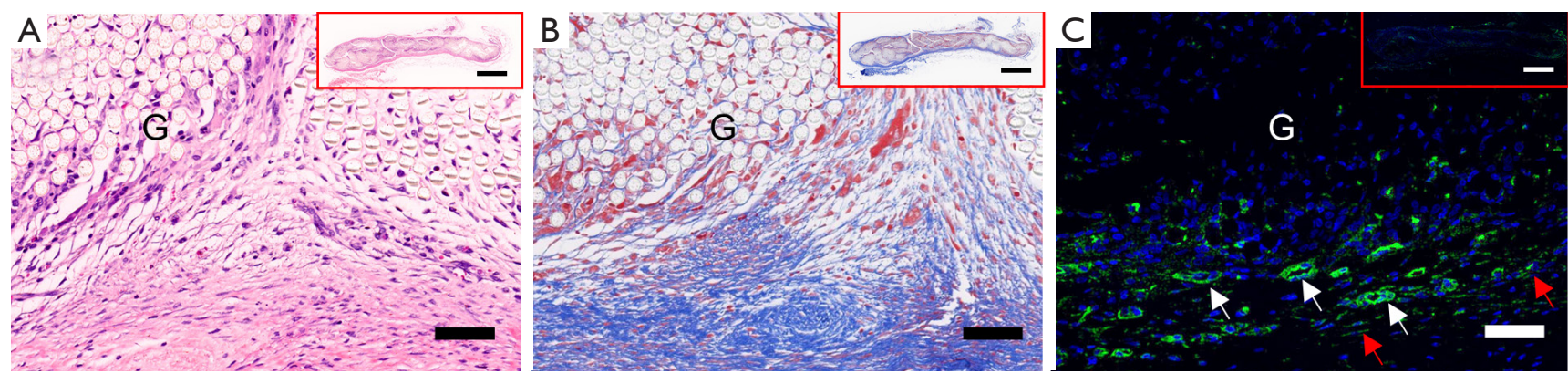

Figure 2 Representative images of the subcutaneously implanted PET by (A) H\&E, (B) Masson Trichrome staining and (C) immunofluorescence of $\alpha$-SMA-positive myofibroblasts (red arrows) and vessels (while arrows) in ATE-PET groups before ACL reconstruction. Bar: $50 \mu \mathrm{m}, 1 \mathrm{~mm}$ (inset). G, graft; ATE, autologous tissue-engineered; PET, polyethylene terephthalate; ACL, anterior cruciate ligament.

variances. According to the normality and homogeneity of variances, the Student's $t$-test or nonparametric MannWhitney U test was used to analyze the differences between the two groups. Data analysis was performed using SPSS 19.0 software (IBM, Chicago, IL, USA), and a two-side P value of $<0.05$ was considered statistically significant.

\section{Results}

\section{The bistology and immunofluorescence of ATE-PET}

As shown in Figure 2, there was obvious cell and tissue ingrowth into the subcutaneously implanted PET graft, indicating that the PET graft was firmly incorporated into the host tissues. Moreover, the expression of $\alpha$-SMA-positive vessels and myofibroblasts was detected around the graft, suggesting adequate pre-vascularization and myofibroblast aggregation of the ligament before ACL reconstruction.

\section{Macroscopic observation of ACL reconstruction}

As shown in Figure 3, there was little synovial coverage of the graft in the PET group, while some synovial tissues with fewer inflammatory reactions were observed in the ATEPET group at 4 weeks after ACL reconstruction. At 12 weeks, there was slight synovial coverage with a progressive inflammatory reaction in the PET group. In contrast, thick tissue coverage with a glossy appearance was observed in the ATE-PET group.

\section{Micro-CT analysis}

At 4 weeks after ACL reconstruction, no significant differences were detected in the BMD and the BV/TV of the femoral and tibial bone tunnels between the PET and ATE-PET groups (Figure 4). At 12 weeks, the ATE-PET group presented significantly higher $\mathrm{BMD}$ in both the femoral tunnel $(\mathrm{P}<0.01)$ and tibial tunnel $(\mathrm{P}=0.01)$ compared with the PET group. Similarly, the BV/TV of both the femoral tunnel $(\mathrm{P}=0.02)$ and tibial tunnel $(\mathrm{P}=0.02)$ were also significantly higher at 12 weeks in the ATE-PET group.

\section{Histological assessment}

Histological assessment was conducted at both the intraosseous and intra-articular parts. Cell infiltration and collagen fiber density increased in the intra-articular part of the graft from week 4 to 12 postoperatively in both groups (Figure 5). However, more collagen fibers growing into the graft with fewer inflammatory reactions were observed in the ATE group. Furthermore, the CVF in the ATE-PET group was significantly greater than that in the PET group at both $4(\mathrm{P}<0.01)$ and 12 weeks $(\mathrm{P}<0.01)$, suggesting better collagen formation and remodeling in the ATE-PET group.

With regard to the intraosseous part of the graft, obvious graft-to-bone interfaces were observed in both groups at 4 weeks with fibrovascular tissue (Figure $6 A, B$ ), and no significant difference was found in terms of average interface width (Figure $6 C, \mathrm{P}=0.45$ ). At 12 weeks, the interface width between the host bone and graft was significantly smaller in the ATE-PET group $(\mathrm{P}<0.01)$, and more collagen fibers infiltrated into the graft.

\section{Immunofluorescence imaging}

At the intra-articular part of the graft, more $\alpha$-SMA- 

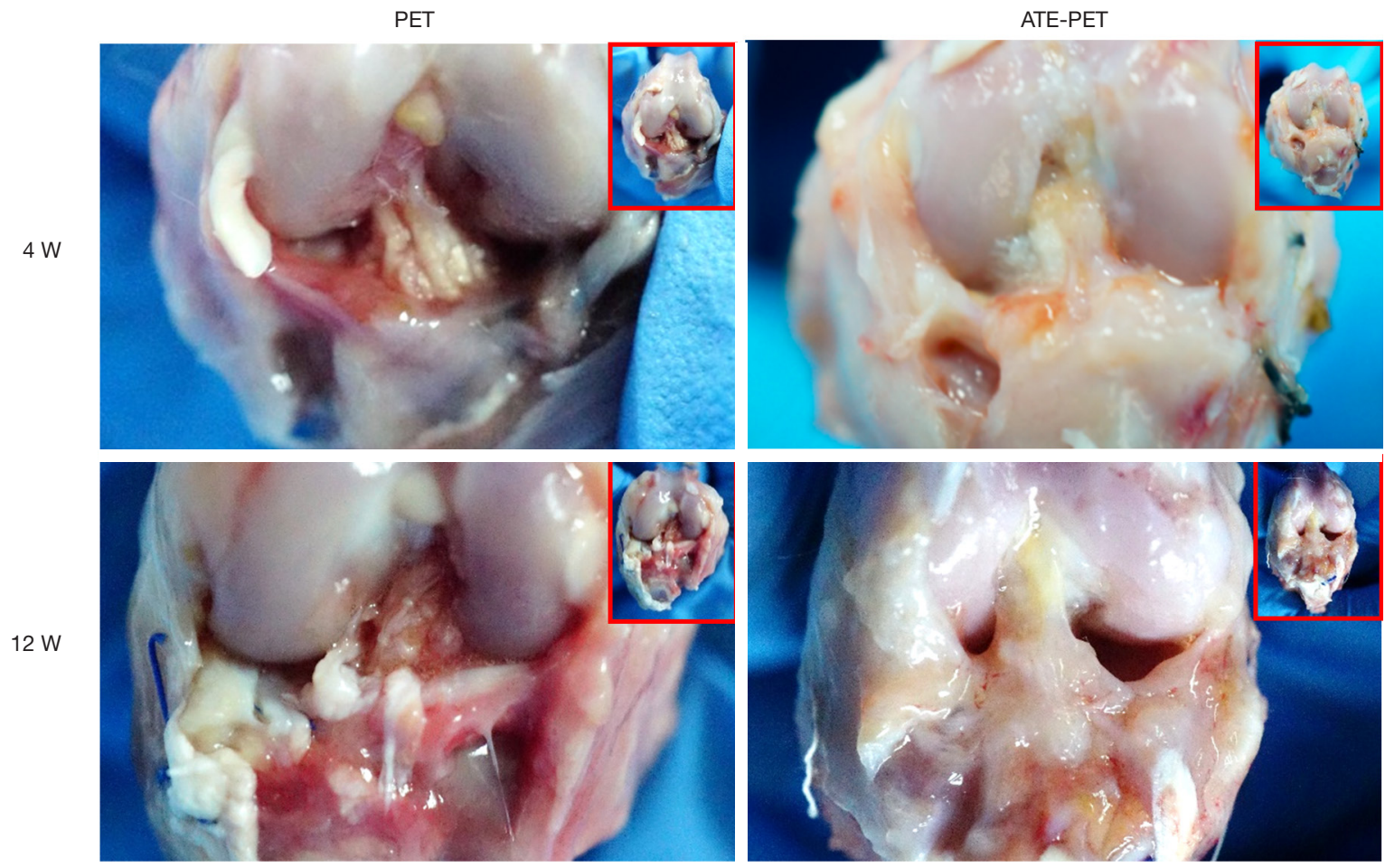

Figure 3 Macroscopic observation of the intra-articular graft in the PET and ATE-PET groups at 4 and 12 weeks after ACL reconstruction. PET, polyethylene terephthalate. ATE, autologous tissue-engineered.

A

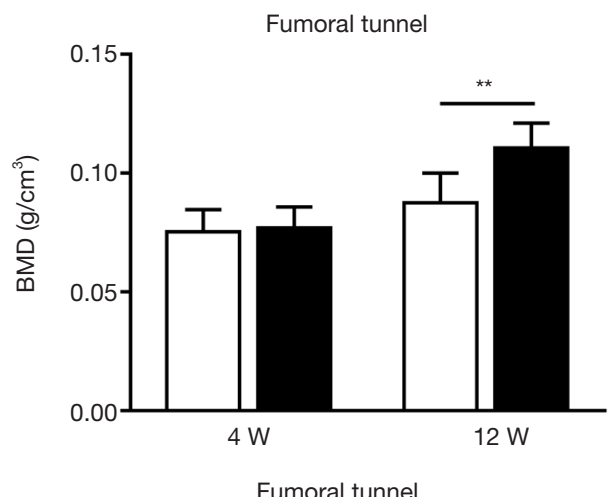

B

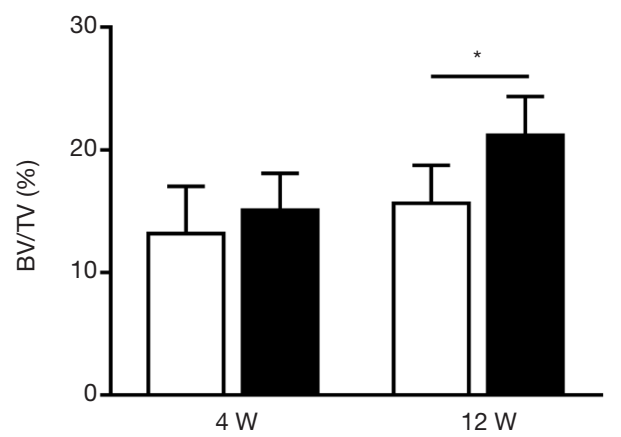

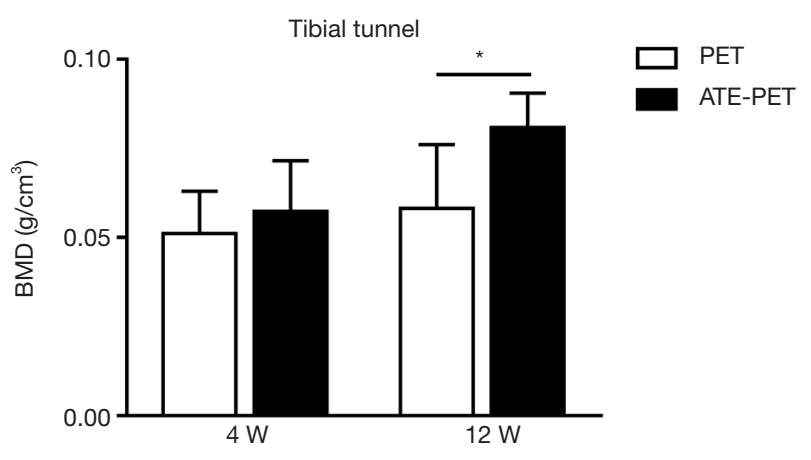

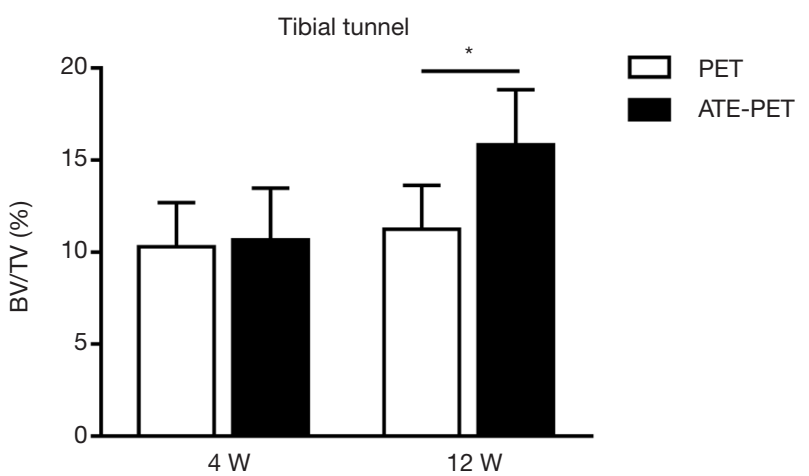

Figure 4 Micro-CT analysis of the bone tunnel at 4 and 12 weeks postoperatively. (A) BMD at the femoral and tibial sides and the (B) BV/TV at the femoral and tibial sides for ACL reconstruction in the PET and ATE-PET groups at 4 and 12 weeks postoperatively. ${ }^{*} \mathrm{P}<0.05,{ }^{* *} \mathrm{P}<0.01$. $\mathrm{BMD}$, bone mineral density; BV/TV, bone volume/total volume ratio; PET, polyethylene terephthalate; ATE, autologous tissue-engineered. 
A

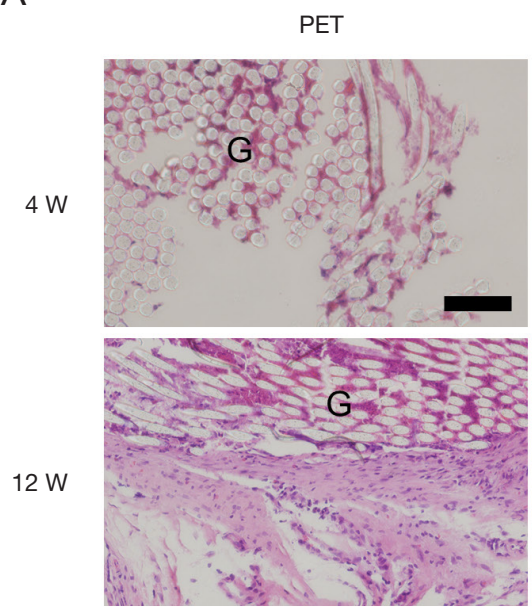

B
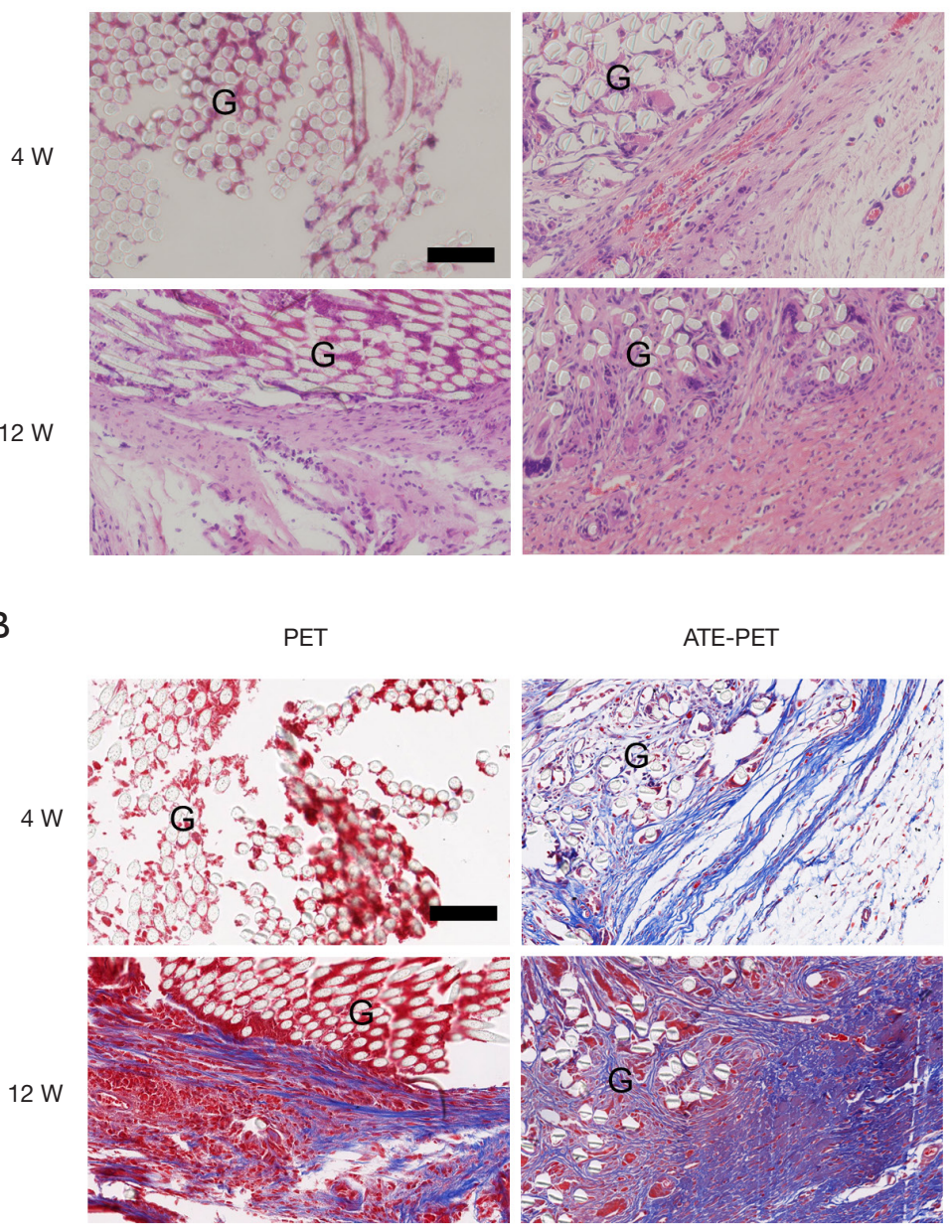

C
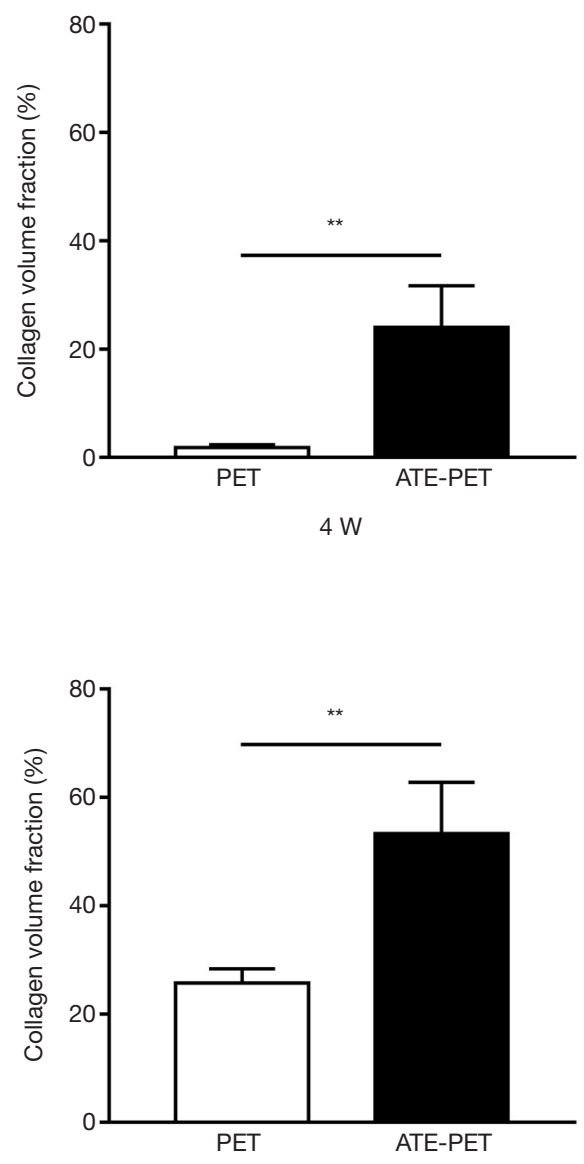

$12 \mathrm{~W}$

Figure 5 Representative images of the intra-articular part of the graft by (A) H\&E staining and (B) Masson Trichrome staining in the PET and ATE-PET groups at 4 and 12 weeks postoperatively; (C) collagen volume fraction of regenerated tissues in the intra-articular part of graft. Bar: $100 \mu \mathrm{m} .{ }^{* *} \mathrm{P}<0.01$. PET, polyethylene terephthalate. ATE, autologous tissue-engineered. G, graft.

positive vessels and $\alpha$-SMA-positive myofibroblasts were observed in the ATE-PET group at 4 weeks postoperatively (both $\mathrm{P}<0.01$ ) (Figure 7). From 4 (early stage) to 12 weeks (late stage), both vessels and myofibroblasts decreased in number in the ATE-PET group and increased in the PET group. Compared with the PET group, the number of $\alpha$-SMA-positive myofibroblasts in the ATE-PET group was significantly lower at 12 weeks postoperatively $(\mathrm{P}<0.01)$.

At the intraosseous part of the graft, the numbers of $\alpha$-SMA-positive vessels and myofibroblasts were significantly greater in the ATE-PET group at 4 weeks (both $\mathrm{P}<0.01$ ) (Figure 8). Moreover, at 12 weeks, the numbers of $\alpha$-SMA-positive vessels and myofibroblasts were significantly smaller in the ATE-PET group than those in the PET group $(\mathrm{P}<0.01)$.

\section{Biomechanical testing}

All specimens failed by pull-out from the bone tunnels, and no graft rupture occurred. As shown in Figure 9, the failure load $(\mathrm{N})$ and stiffness $(\mathrm{N} / \mathrm{mm})$ were not significantly different in the ATE-PET and PET groups at 4 weeks postoperatively $(19.9 \pm 3.6$ vs. $19.2 \pm 2.4 \mathrm{~N}, \mathrm{P}=0.71 ; 7.1 \pm 1.2$ vs. $7.0 \pm 0.9 \mathrm{~N} / \mathrm{mm}, \mathrm{P}=0.81$, respectively). However, at 12 weeks, the ATE-PET group presented significantly greater failure load and stiffness than the PET group (53.7 \pm 5.4 vs. $42.5 \pm 4.5 \mathrm{~N}, \mathrm{P}<0.01 ; 12.9 \pm 3.0$ vs. $9.8 \pm 1.3 \mathrm{~N} / \mathrm{mm}$, $\mathrm{P}=0.04$, respectively). 
A

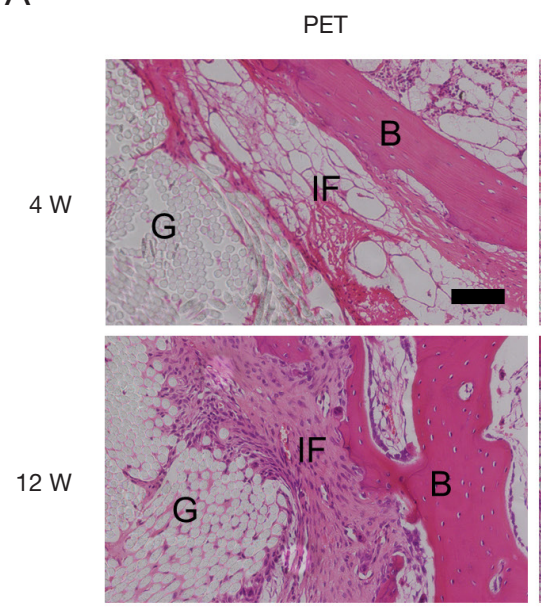

B

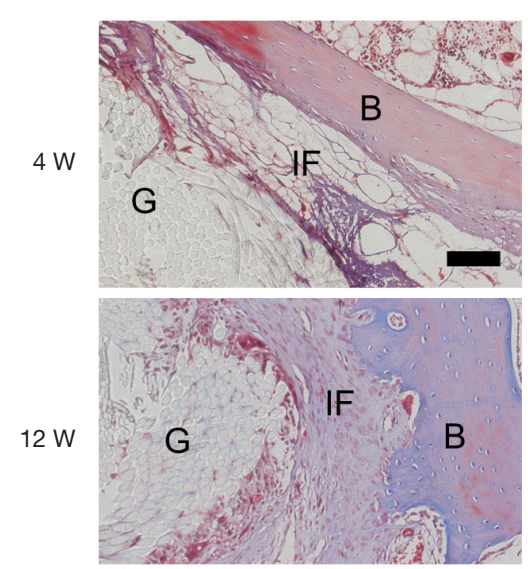

ATE-PET
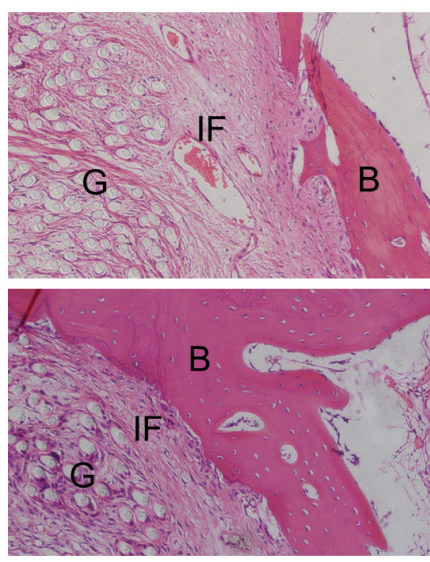

ATE-PET

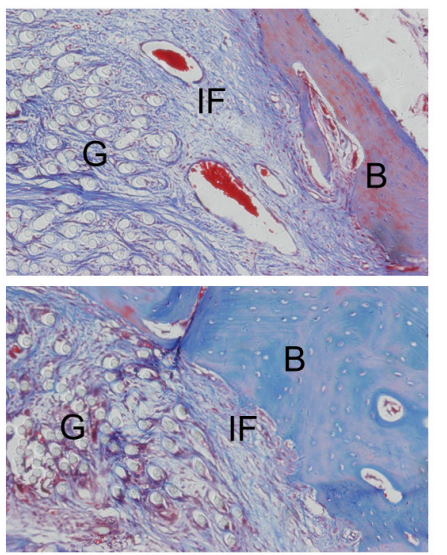

C
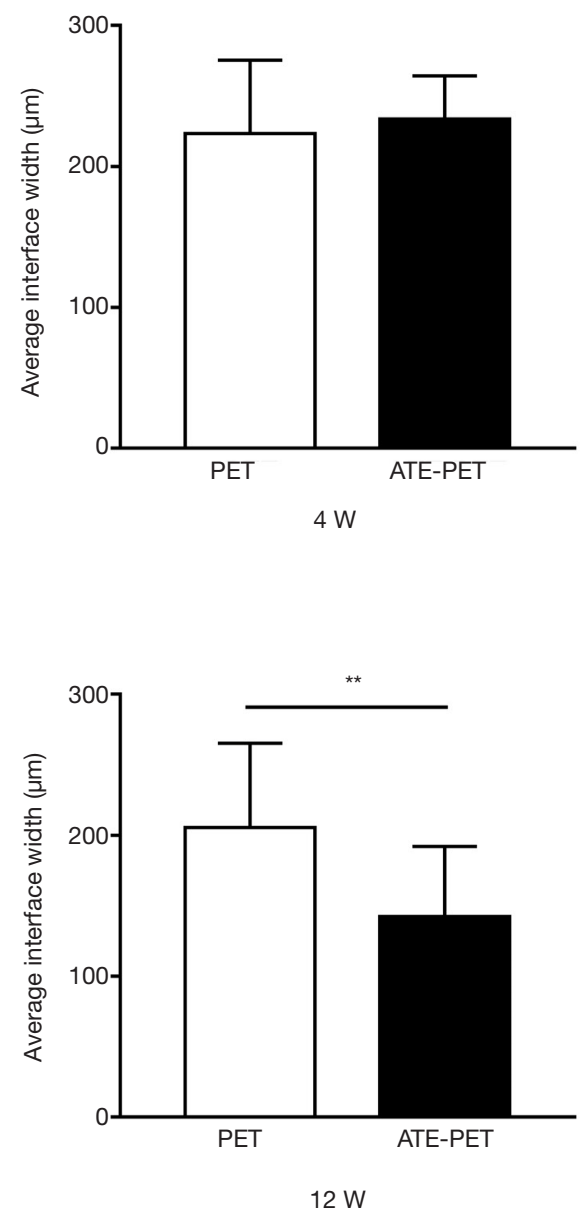

Figure 6 Representative images of the intraosseous part of graft by (A) H\&E staining and (B) Masson Trichrome staining in the PET and ATE-PET groups at 4 and 12 weeks postoperatively; (C) quantification of the interface width between the bone and graft. Bar: $100 \mu \mathrm{m}$. ${ }^{* *} \mathrm{P}<0.01$. PET, polyethylene terephthalate; ATE, autologous tissue-engineered; IF, interface. B, bone, G, graft.

\section{Discussion}

The main disadvantage of the PET artificial ligament is its hydrophobicity, which is unfavorable for tissue ingrowth and graft vascularization, leading to poor incorporation into the host. In the present study, with a subcutaneous implantation preprocess before ACL reconstruction, the ATE-PET graft was highly pre-vascularized, along with myofibroblast adhesion and collagen fiber ingrowth. It was demonstrated that the ATE-PET graft accelerated graft ligamentization and osseointegration processes after ACL reconstruction.

With regard to the intra-articular part of the graft, the positive effect of autologous tissue engineering on graft ligamentization was identified. More collagen infiltration was found at both 4 and 12 weeks, which was beneficial for graft ligamentization. Furthermore, in the analysis of the intraosseous part of the graft, the interface width between the graft and bone in the ATE-PET group was significantly smaller than that in the PET group at 12 weeks, suggesting that the bone tunnel enlargement was antagonized in the ATE-PET group after ACL reconstruction compared with that in the PET group. Meanwhile, with the quantification of graft osseointegration in the bone tunnel by micro-CT, it was proven that the $\mathrm{BMD}$ and $\mathrm{BV} / \mathrm{TV}$ around both femoral and tibial tunnels in the ATE-PET group were significantly higher than those in the PET group. Owing to the superiority in both intra-articular graft ligamentization and 
A
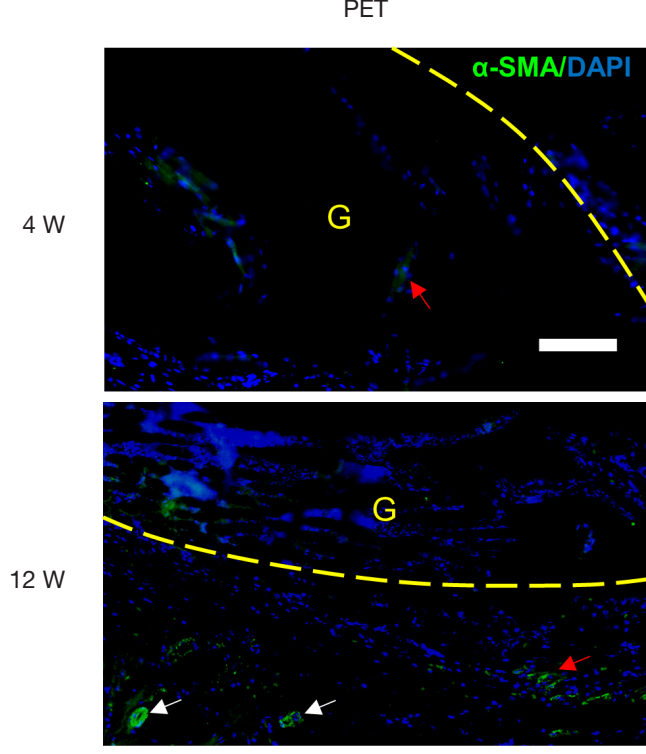

B

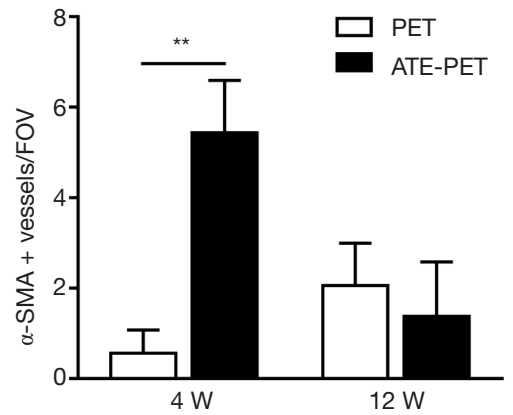

ATE-PET
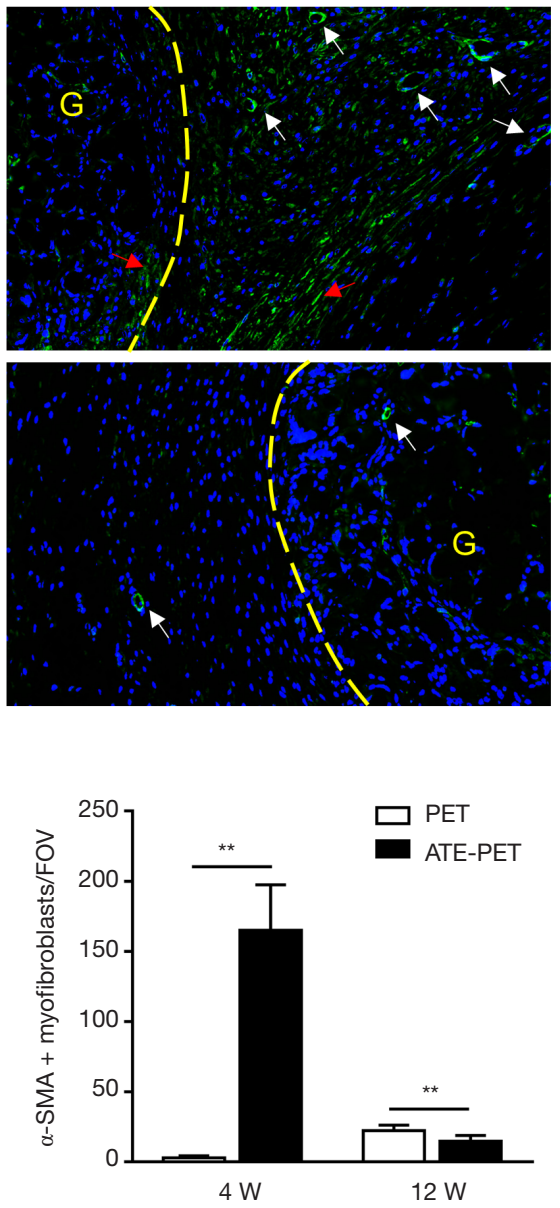

Figure 7 Immunofluorescence imaging and quantification of $\alpha$-SMA expression at the intra-articular part of the graft at 4 and 12 weeks postoperatively. (A) Representative images of the intra-articular part of the graft by immunofluorescence of $\alpha$-SMA-positive myofibroblasts (red arrows) and vessels (white arrows) in the PET and ATE-PET groups at 4 and 12 weeks postoperatively. (B) Quantification of the $\alpha$-SMA-positive vessels and myofibroblasts. Bar: $100 \mu \mathrm{m} .{ }^{* *} \mathrm{P}<0.01$. PET, polyethylene terephthalate. ATE, autologous tissue-engineered. $\alpha$-SMA, $\alpha$-smooth muscle actin. G, graft. FOV, field of view.

intraosseous graft osseointegration, the ATE-PET group showed better biomechanical performance in failure load and stiffness than the PET group at 12 weeks after surgery.

It is widely acknowledged that successful ligamentization and osseointegration processes after ACL reconstruction are markedly difficult, and these are associated with the early inflammatory response of the micro-environment and hostversus-graft reaction (29-31). In addition, inflammatory reactions contribute to the degradation of the extracellular matrix (ECM) within the healing site, thus affecting graft incorporation and remodeling (31-33). We performed subcutaneous implantation of the PET graft to avoid the early inflammatory response of the host-versus-graft in both the bone tunnel and intra-articular environment. As a result, the ATE-PET group showed obviously less inflammatory reaction on macroscopic observation after ACL reconstruction. Moreover, we observed better cell ingrowth and tissue infiltration along with more ECM accumulation around the graft on histological assessment, suggesting that the subcutaneous implantation preprocess could effectively reduce inflammation during the early healing phase and thus accelerate the graft incorporation and remodeling processes.

Adequate vascularization is beneficial for the delivery of nutrients and oxygen for cell ingrowth and matrix accumulation during the early phase after 
A
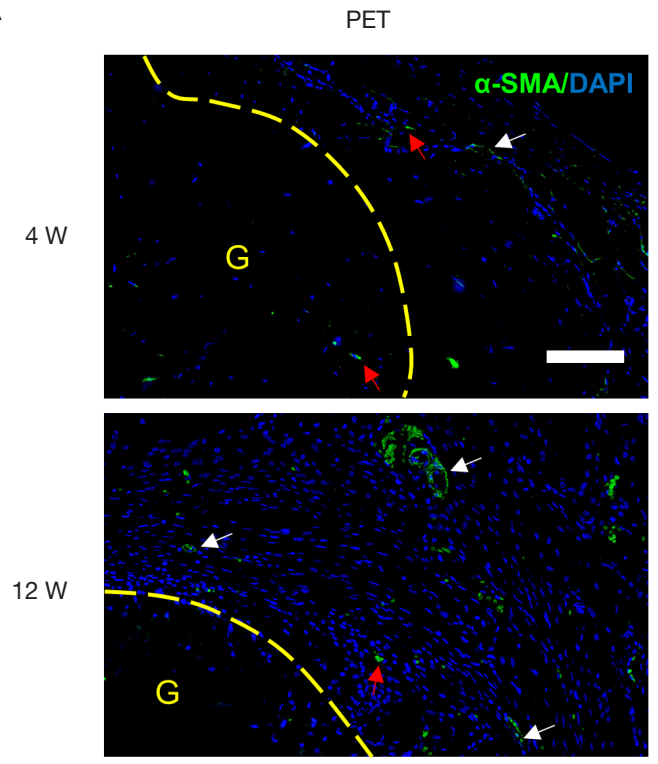

B

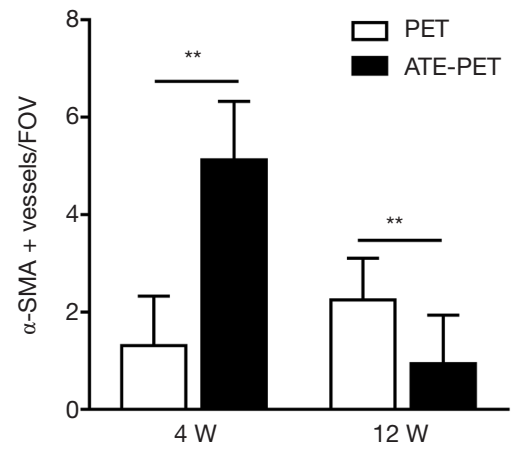

ATE-PET
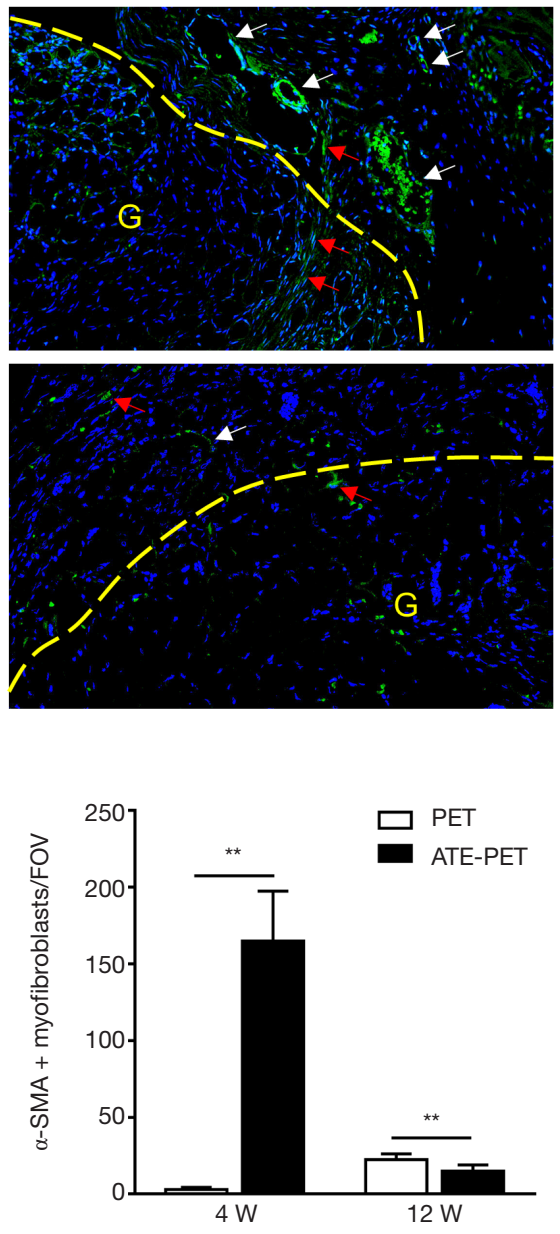

Figure 8 Immunofluorescence imaging and quantification of $\alpha$-SMA expression at the intraosseous part of the graft at 4 and 12 weeks postoperatively. (A) Representative images of the intraosseous part of the graft by immunofluorescence of $\alpha$-SMA-positive myofibroblasts at the interface (red arrows) and vessels (white arrows) in PET and ATE-PET groups at 4 and 12 weeks postoperatively. (B) Quantification of the $\alpha$-SMA-positive vessels and myofibroblast cells. Bar: $100 \mu \mathrm{m} .{ }^{* *} \mathrm{P}<0.01$. PET, polyethylene terephthalate. ATE, autologous tissueengineered. G, graft. $\alpha$-SMA, $\alpha$-smooth muscle actin. FOV, field of view.

ACL reconstruction, which plays a vital role in graft ligamentization and osseointegration. Liu et al. reported that a hamstring tendon graft with intact tibial insertion could preserve enough blood supply in the early stage, and such a vascularized graft promoted the ligamentization and osseointegration processes after ACL reconstruction in a rabbit model (34). Despite such a positive effect, overstimulation of vascularization at the late healing stage is not appropriate, as it may cause pathological fibrosis (35-38). "Pre-vascularization," as a newly emerged concept in tissue engineering, emphasizes the pre-formed functional vascular networks with grafts or scaffolds embedded prior to their implantation, which enhances the graft connection to the host vascular system (22). The novelty of our study lies in the fact that a PET graft, with preliminary subcutaneous implantation, underwent an autologous tissue engineering process and became highly pre-vascularized. When ACL reconstruction was performed with this pre-vascularized graft, the $\alpha$-SMA-positive vessels in both the intra-articular and intraosseous parts were significantly greater in the ATE-PET group than in the PET group at 4 weeks, suggesting more blood supply during the early healing phase. Furthermore, the number of $\alpha$-SMA-positive vessels markedly declined at 12 weeks in the ATE-PET group, 
A

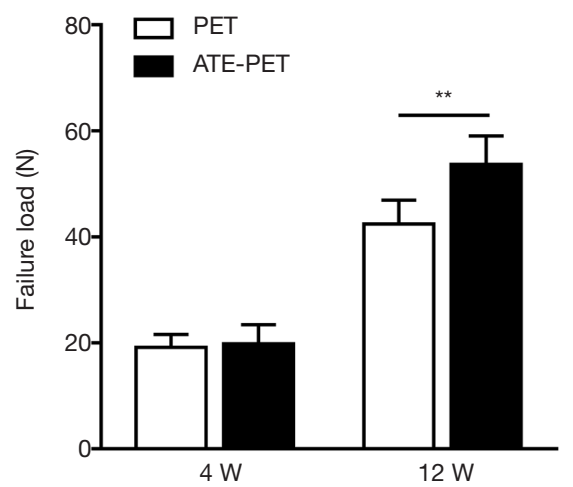

B

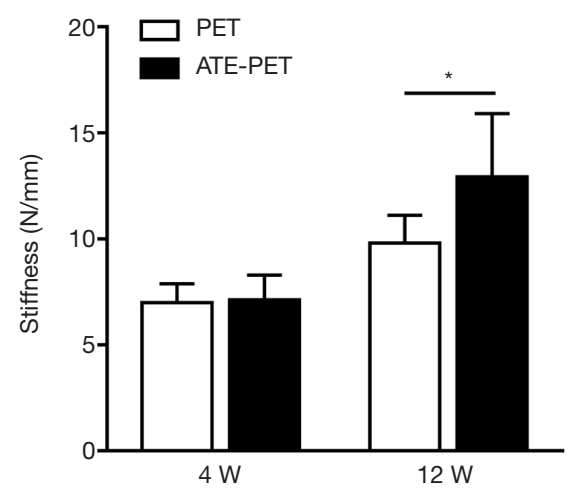

Figure 9 Biomechanical results at 4 and 12 weeks postoperatively. (A) Failure load and (B) stiffness of the femur-graft-tibia complex after ACL reconstruction at 4 and 12 weeks postoperatively in the PET and ATE-PET groups. ${ }^{*} \mathrm{P}<0.05 ;{ }^{* *} \mathrm{P}<0.01$. PET, polyethylene terephthalate. ATE, autologous tissue-engineered.

indicating that vascularization receded, which, to some extent, prevented fibrogenesis and scar formation at the healing site.

Moreover, $\alpha$-SMA-positive myofibroblasts exist in the normal ligaments and tendons (39) and play an important role in the healing process after ACL reconstruction. Myofibroblasts contributed to collagen production and fiber bundle formation at the early phase of graft remodeling $(27,39)$ and helped organize Sharpey-like fibers at the graft-to-bone interface (27). In our study, the ATE-PET graft was populated with myofibroblasts before ACL reconstruction. The level of $\alpha$-SMA-positive myofibroblasts was significantly higher at the graft-to-bone interface in the ATE-PET group in the early stage (4 weeks after surgery) than in the PET group. However, at 12 weeks after surgery, the number of $\alpha$-SMA-positive myofibroblasts decreased in the ATE-PET group and was significantly lower than that in the PET group. Analogously, the biological trend of the number of $\alpha$-SMA-positive myofibroblasts around the intraarticular part of the graft was consistent with that at the intraosseous part during the healing process. Sun et al. also found similar fluctuations in $\alpha$-SMA-positive myofibroblasts, which facilitated the ligamentization and osseointegration processes after ACL reconstruction with autografts in a rat model (27). In our study, myofibroblasts also had a positive effect on graft ligamentization and osseointegration. As two major functions of fibroblasts are to establish the ECM and to remodel the graft, this fluctuation might indicate that the differentiation and proliferation of myofibroblasts first participate in tissue regeneration and then withdraw after successful ECM remodeling.
The study was not without limitations. First, a rabbit ACL reconstruction model is a proof-of-concept model that may not fully simulate human physiological environments and mechanical conditions. Large animal models, such as pigs or canines, are needed for further evaluation before clinical trials. Furthermore, a longer observation time is needed to further evaluate the effects of the ATE-PET graft. Finally, whether the subcutaneous implantation preprocess of the PET graft will cause side effects at the implanted site, such as pain and dysfunction, needs to be identified.

\section{Conclusions}

This study developed an ATE-PET artificial ligament with pre-vascularization and myofibroblast aggregation. This could effectively accelerate intra-articular graft ligamentization and intraosseous graft osseointegration, thus enhancing the biomechanical properties after ACL reconstruction in a rabbit model. Therefore, the autologous tissue engineering preprocess could be considered as a potential strategy to improve clinical outcomes and decrease the failure rate of artificial ligaments during ACL reconstruction.

\section{Acknowledgments}

Funding: This study was supported by the National Key Research and Development Program of China (Grant No. 2018YFC1106200 and 2018YFC1106202), the National Natural Science Foundation of China (Grant No. 81871753 and 81772341), the Western Medicine Guidance Project of Science and Technology Commission of Shanghai 
Municipality of China (Grant No. 17411966400), China Postdoctoral Science Foundation (Grant No. 2020M671154) and the Open Foundation of State Key Laboratory of Molecular Engineering of Polymers (Grant No. K2021-23).

\section{Footnote}

Reporting Checklist: The authors have completed the ARRIVE reporting checklist. Available at http://dx.doi. org/10.21037/atm-20-8048

Data Sharing Statement: Available at http://dx.doi. org/10.21037/atm-20-8048

Conflicts of Interest: All authors have completed the ICMJE uniform disclosure form (available at http://dx.doi. org/10.21037/atm-20-8048). The authors have no conflicts of interest to declare.

Ethical Statement: The authors are accountable for all aspects of the work in ensuring that questions related to the accuracy or integrity of any part of the work are appropriately investigated and resolved. All animal experiments were approved by the Animal Care and Experiment Committee of Shanghai Jiao Tong University (No.: 201018) and were conducted in accordance with the local animal care guidelines.

Open Access Statement: This is an Open Access article distributed in accordance with the Creative Commons Attribution-NonCommercial-NoDerivs 4.0 International License (CC BY-NC-ND 4.0), which permits the noncommercial replication and distribution of the article with the strict proviso that no changes or edits are made and the original work is properly cited (including links to both the formal publication through the relevant DOI and the license). See: https://creativecommons.org/licenses/by-nc-nd/4.0/.

\section{References}

1. Ahmad CS, Gardner TR, Groh M, et al. Mechanical properties of soft tissue femoral fixation devices for anterior cruciate ligament reconstruction. Am J Sports Med 2004;32:635-40.

2. LaBella CR, Hennrikus W, Hewett TE. Anterior cruciate ligament injuries: diagnosis, treatment, and prevention. Pediatrics 2014;133:e1437-50.

3. Chen T, Zhang P, Chen J, et al. Long-Term Outcomes of Anterior Cruciate Ligament Reconstruction Using Either
Synthetics With Remnant Preservation or Hamstring Autografts: A 10-Year Longitudinal Study. Am J Sports Med 2017;45:2739-50.

4. Batty LM, Norsworthy CJ, Lash NJ, et al. Synthetic devices for reconstructive surgery of the cruciate ligaments: a systematic review. Arthroscopy 2015;31:957-68.

5. Schindler OS. Surgery for anterior cruciate ligament deficiency: a historical perspective. Knee Surg Sports Traumatol Arthrosc 2012;20:5-47.

6. Smith BD, Grande DA. The current state of scaffolds for musculoskeletal regenerative applications. Nat Rev Rheumatol 2015;11:213-22.

7. Cai J, Ai C, Chen J, et al. Biomineralizaion of hydroxyapatite on polyethylene terephthalate artificial ligaments promotes graft-bone healing after anterior cruciate ligament reconstruction: An in vitro and in vivo study. J Biomater Appl 2020;35:193-204.

8. Tulloch SJ, Devitt BM, Porter T, et al. Primary ACL reconstruction using the LARS device is associated with a high failure rate at minimum of 6-year follow-up. Knee Surg Sports Traumatol Arthrosc 2019;27:3626-32.

9. Iliadis DP, Bourlos DN, Mastrokalos DS, et al. LARS Artificial Ligament Versus ABC Purely Polyester Ligament for Anterior Cruciate Ligament Reconstruction. Orthop J Sports Med 2016;4:2325967116653359.

10. Guidoin MF, Marois Y, Bejui J et al. Analysis of retrieved polymer fiber based replacements for the ACL. Biomaterials 2000;21:2461-74.

11. Cai J, Wan F, Dong Q, et al. Silk fibroin and hydroxyapatite segmented coating enhances graft ligamentization and osseointegration processes of the polyethylene terephthalate artificial ligament in vitro and in vivo. J Mater Chem B 2018;6:5738-49.

12. Cai J, Zhang Q, Chen J, et al. Electrodeposition of calcium phosphate onto polyethylene terephthalate artificial ligament enhances graft-bone integration after anterior cruciate ligament reconstruction. Bioact Mater 2020;6:783-93.

13. Cai J, Li Zhang, Chen J, et al. Silk fibroin coating through EDC/NHS crosslink is an effective method to promote graft remodeling of a polyethylene terephthalate artificial ligament. J Biomater Appl 2019;33:1407-14.

14. Li H, Li J, Jiang J, et al. An osteogenesis/angiogenesisstimulation artificial ligament for anterior cruciate ligament reconstruction. Acta Biomater 2017;54:399-410.

15. Vaquette C, Viateau V, Guérard S, et al. The effect of polystyrene sodium sulfonate grafting on polyethylene terephthalate artificial ligaments on in vitro mineralisation and in vivo bone tissue integration. Biomaterials 2013;34:7048-63. 
16. Cai J, Wan F, Ai C, et al. The Effect of Remnant Preservation on Tibial Tunnel Enlargement in Anterior Cruciate Ligament Reconstruction with Polyethylene Terephthalate Artificial Ligament in a Large Animal Model. J Knee Surg 2019;32:1094-101.

17. Dai Z, Bao W, Li S, et al. Enhancement of Polyethylene Terephthalate Artificial Ligament Graft Osseointegration using a Periosteum Patch in a Goat Model. Int J Sports Med 2016;37:493-9.

18. Wang XM, Ji G, Wang XM, et al. Biological and Biomechanical Evaluation of Autologous Tendon Combined with Ligament Advanced Reinforcement System Artificial Ligament in a Rabbit Model of Anterior Cruciate Ligament Reconstruction. Orthop Surg 2018;10:144-51.

19. Nau T, Teuschl A. Regeneration of the anterior cruciate ligament: Current strategies in tissue engineering. World J Orthop 2015;6:127-36.

20. Ran J, Hu Y, Le H, et al. Ectopic tissue engineered ligament with silk collagen scaffold for ACL regeneration: A preliminary study. Acta Biomater 2017;53:307-17.

21. Amirsadeghi A, Jafari A, Eggermont LJ, et al. Vascularization strategies for skin tissue engineering. Biomater Sci 2020;8:4073-94.

22. Smirani R, Rémy M, Devillard R, et al. Engineered Prevascularization for Oral Tissue Grafting: A Systematic Review. Tissue Eng Part B Rev 2020;26:383-98.

23. Ai C, Sheng D, Chen J, et al. Surface modification of vascular endothelial growth factor-loaded silk fibroin to improve biological performance of ultra-high-molecularweight polyethylene via promoting angiogenesis. Int J Nanomedicine 2017;12:7737-50.

24. Ma CB, Kawamura S, Deng XH, et al. Bone morphogenetic proteins-signaling plays a role in tendonto-bone healing: a study of rhBMP-2 and noggin. Am J Sports Med 2007;35:597-604.

25. Zhou W, Zi L, Cen Y, et al. Copper Sulfide NanoparticlesIncorporated Hyaluronic Acid Injectable Hydrogel With Enhanced Angiogenesis to Promote Wound Healing. Front Bioeng Biotechnol 2020;8:417.

26. Liu J, Qin Y, Wu Y, et al. The surrounding tissue contributes to smooth muscle cells' regeneration and vascularization of small diameter vascular grafts. Biomater Sci 2019;7:914-25.

27. Sun Y, Chen W, Hao Y, et al. Stem Cell-Conditioned Medium Promotes Graft Remodeling of Midsubstance and Intratunnel Incorporation After Anterior Cruciate Ligament Reconstruction in a Rat Model. Am J Sports Med 2019;47:2327-37.

28. Makarevich PI, Boldyreva MA, Gluhanyuk EV, et al.
Enhanced angiogenesis in ischemic skeletal muscle after transplantation of cell sheets from baculovirus-transduced adipose-derived stromal cells expressing VEGF165. Stem Cell Res Ther 2015;6:204.

29. Chen X, Qi YY, Wang LL, et al. Ligament regeneration using a knitted silk scaffold combined with collagen matrix. Biomaterials 2008;29:3683-92.

30. Shen W, Chen X, Hu Y, et al. Long-term effects of knitted silk-collagen sponge scaffold on anterior cruciate ligament reconstruction and osteoarthritis prevention. Biomaterials 2014;35:8154-63.

31. Janssen RPA, Scheffler SU. Intra-articular remodelling of hamstring tendon grafts after anterior cruciate ligament reconstruction. Knee Surg Sports Traumatol Arthrosc 2014;22:2102-8.

32. Murray MM, Spindler KP, Ballard P, et al. Enhanced histologic repair in a central wound in the anterior cruciate ligament with a collagen-platelet-rich plasma scaffold. J Orthop Res 2007;25:1007-17.

33. Murray MM. Current status and potential of primary ACL repair. Clin Sports Med 2009;28:51-61.

34. Liu S, Sun Y, Wan F, et al. Advantages of an Attached Semitendinosus Tendon Graft in Anterior Cruciate Ligament Reconstruction in a Rabbit Model. Am J Sports Med 2018;46:3227-36.

35. Armulik A, Genové G, Betsholtz C. Pericytes: developmental, physiological, and pathological perspectives, problems, and promises. Dev Cell 2011;21:193-215.

36. Cappellari O, Cossu G. Pericytes in development and pathology of skeletal muscle. Circ Res 2013;113:341-7.

37. Wong SP, Rowley JE, Redpath AN, et al. Pericytes, mesenchymal stem cells and their contributions to tissue repair. Pharmacol Ther 2015;151:107-20.

38. Worthley DL, Churchill M, Compton JT, et al. Gremlin 1 identifies a skeletal stem cell with bone, cartilage, and reticular stromal potential. Cell 2015;160:269-84.

39. Weiler A, Unterhauser FN, Bail HJ, et al. Alpha-smooth muscle actin is expressed by fibroblastic cells of the ovine anterior cruciate ligament and its free tendon graft during remodeling. J Orthop Res 2002;20:310-7.

Cite this article as: Cai $\mathrm{J}, \mathrm{Xu} \mathrm{J}$, Kang Y, Li Y, Wang L, Yan X, Jiang J, Zhao J. Acceleration of ligamentization and osseointegration processes after anterior cruciate ligament reconstruction with autologous tissue-engineered polyethylene terephthalate graft. Ann Transl Med 2021;9(9):770. doi: 10.21037/atm-20-8048 